University of Nebraska - Lincoln

DigitalCommons@University of Nebraska - Lincoln

Faculty Publications, Department of Psychology

Psychology, Department of

4-1-2006

\title{
Forensic interviewing in child sexual abuse cases: Current techniques and future directions
}

\author{
Lindsay E. Cronch \\ University of Nebraska-Lincoln, lecronch@yahoo.com \\ Jodi L. Viljoen \\ University of Nebraska-Lincoln, jviljoen@sfu.ca \\ David J. Hansen \\ Univertsity of Nebraska-Lincoln, dhansen1@unl.edu
}

Follow this and additional works at: https://digitalcommons.unl.edu/psychfacpub

Part of the Psychiatry and Psychology Commons

Cronch, Lindsay E.; Viljoen, Jodi L.; and Hansen, David J., "Forensic interviewing in child sexual abuse cases: Current techniques and future directions" (2006). Faculty Publications, Department of Psychology. 6.

https://digitalcommons.unl.edu/psychfacpub/6

This Article is brought to you for free and open access by the Psychology, Department of at DigitalCommons@University of Nebraska - Lincoln. It has been accepted for inclusion in Faculty Publications, Department of Psychology by an authorized administrator of DigitalCommons@University of Nebraska - Lincoln. 
Aggression

And

Violent

Behavior

\title{
Forensic interviewing in child sexual abuse cases: Current techniques and future directions
}

\author{
Lindsay E. Cronch, Jodi L. Viljoen and David J. Hansen \\ University of Nebraska-Lincoln, USA \\ Submitted 22 July 2005; accepted 27 July 2005. Available online 8 September 2005.
}

\begin{abstract}
In child sexual abuse cases, skillful forensic interviews are important to ensure the protection of innocent individuals and the conviction of perpetrators. Studies have examined several factors that influence disclosure during interviews, including both interviewer and child characteristics. Numerous interviewing techniques have received attention in the literature, including allegation blind interviews, open-ended questioning, cognitive interviewing, the Touch Survey, truth-lie discussions, and anatomical dolls. Recent studies have examined new directions in forensic interviewing, such as structured interview protocols and the extended forensic evaluation model. In addition, the child advocacy center model has been established as a strategy to prevent repeated interviewing. Child Advocacy Centers provide a safe, child-friendly atmosphere for children and families to receive services. Limitations of the research are discussed and empirically based recommendations for interviewers are provided.
\end{abstract}

Keywords: Sexual abuse; Forensic interviews; Children

\section{Contents}

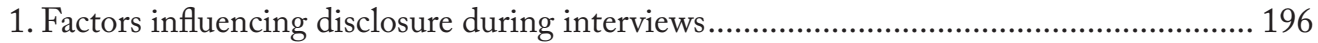

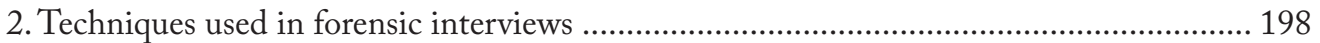

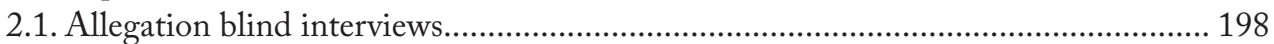

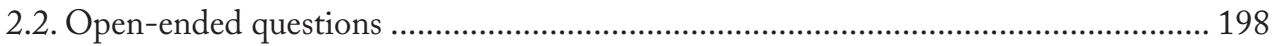

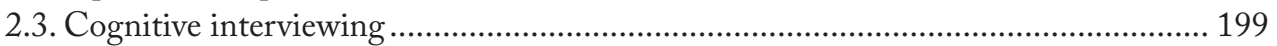

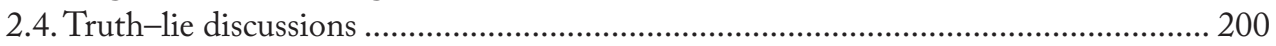

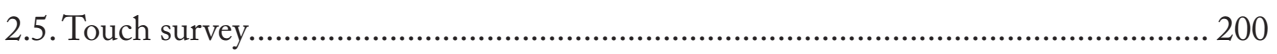

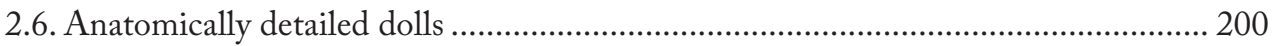

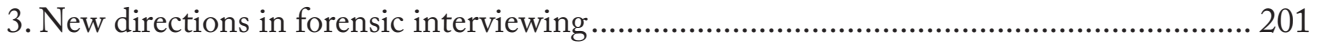

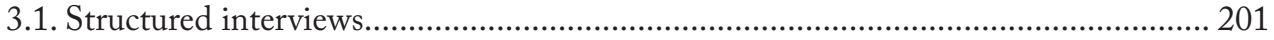

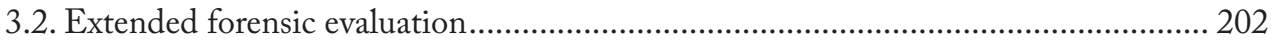

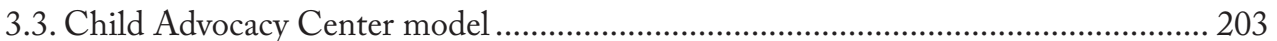

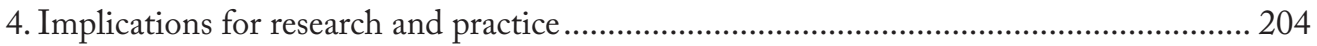

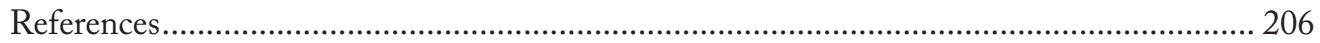

Published in Aggression and Violent Behavior 11:3 (May-June 2006), pp. 195-207. doi:10.1016/j.avb.2005.07.009 Copyright $\odot 2005$ Elsevier Ltd. Used by permission. 
Child sexual abuse is an alarmingly prevalent problem in the United States. According to reports from child protective service agencies, 78,188 children were sexually abused in 2003 at the rate of 1.2 per 1000 children (U.S. Department of Health and Human Services, 2005). These numbers represent only substantiated cases of abuse, and it is commonly assumed that actual rates of sexual abuse are most certainly much greater. Failure to substantiate and underreporting have led to gross underestimates of the incidence of sexual abuse (Hsu et al., 2002 and Tyler, 2002). Furthermore, of the children with substantiated sexual abuse cases in 2003, only 4\% were actually removed from the home (U.S. Department of Health and Human Services, 2005). These statistics are unsettling, in light of research suggesting that a history of sexual abuse greatly increases the risk for future revictimization (e.g., Boney-McCoy \& Finkelhor, 1995). For these reasons, skillful forensic interviews in child sexual abuse cases are extremely important in ensuring that victims and falsely accused individuals are protected and perpetrators are convicted.

According to the American Professional Society on the Abuse of Children (APSAC), the purpose of the forensic interview is "to elicit as complete and accurate a report from the alleged child or adolescent victim as possible in order to determine whether the child or adolescent has been abused (or is in imminent risk of abuse) and, if so, by whom" (APSAC, 2002, p. 2). Interviews are typically conducted by law enforcement officers, child protective services personnel, or specialized forensic interviewers, although medical and mental health professionals often participate as well (APSAC, 2002, Carnes, 2000, Lanning, 2002, National Children's Advocacy Center, 2005a and National Children's Advocacy Center, 2005b). As demonstrated by highly publicized cases, such as that of Kelly Michaels and the abuse allegations involving her daycare center (Bruck \& Ceci, 1995), bad interviewing can lead to serious consequences. These may include eliciting false allegations, putting children and families through unnecessary stress, decreasing a child victim's credibility in court, contaminating facts, reducing probability of conviction, draining resources through unsuccessful trials and investigations, and reducing resources available for legitimate abuse cases (Wood \& Garven, 2000). To avoid these negative outcomes, current interviewing techniques must be continuously examined and revised as necessary. The purpose of this paper is to examine the current techniques used in forensic interviews with child sexual abuse victims, as well as new directions in research and practice. Empirically based recommendations for interviewers will be discussed.

\section{Factors influencing disclosure during interviews}

Children are understandably reluctant to disclose information about abuse. Sexual abuse is often a very private, embarrassing, and shameful topic to discuss and many children are unlikely to ever tell their story (Hsu et al., 2002 and Tyler, 2002). For these reasons, it is important that research examine barriers to disclosure and factors that are likely to improve disclosure rates during forensic interviews. Several factors that appear to influence the disclosure of sexual abuse have been explored in the literature. These factors include individual characteristics of the interviewer (i.e., gender), the child or adolescent (i.e., age), and the interview itself.

The interviewer carries enormous responsibility in child sexual abuse cases, as he or she can single-handedly determine the probability of disclosure and, thereby, the likelihood of prosecution. An interviewer has the power to elicit false allegations (e.g., Bruck \& Ceci, 1995, Lamb \& Fauchier, 2001, Saywitz et al., 2002 and Wood \& Garven, 2000), to determine accuracy and amount of details provided by the victim (e.g., Davies et al., 2000, Hershkowitz et al., 2002, Lamb \& Garretson, 2003, Sternberg et al., 1996 and Wood \& Garven, 2000), and to prevent the victim from disclosing altogether (e.g., Saywitz et al., 2002 and Wood \& Garven, 2000). The interviewer's influence may stem from personal characteristics, but is often a function of interviewing skill. Wood and Garven (2000) suggest that a distinction be made between improper interviewing and clumsy interviewing. The authors define improper interviewing as the use of techniques that research has shown to be risky and ineffective. Four categories of improper interviewing techniques are described, including use of reinforcement (i.e., punishments and rewards), social influence (i.e., telling the child what others have said), asking suggestive or leading questions (i.e., introducing information that the child has not disclosed), and removing the child from 
direct experience (i.e., asking what might have happened). These techniques are likely to lead to negative consequences, such as false allegations and reduced likelihood of conviction (e.g., Bruck \& Ceci, 1995 and Wood \& Garven, 2000).

On the other hand, clumsy interviewing is defined by Wood and Garven (2000) as failure to use recommended interviewing techniques. Clumsy interviews may occur even with highly trained interviewers, as a result of forgetfulness, lack of skill, and lack of supervision. Consequences of clumsy interviewing may include lack of detail in children's responses, reduced credibility of children's statements, and reduced likelihood of conviction. Wood and Garven recommend that law enforcement personnel and caseworkers be trained to recognize and avoid using improper interviewing techniques. Furthermore, supervision is highly beneficial in reducing improper and clumsy interviewing (e.g., Lamb, Sternberg, Orbach, Esplin, \& Mitchell, 2002). Interviews should be taped, and interview transcripts and tapes should be regularly reviewed by supervisors. To avoid improper and clumsy interviewing, certain interviewer qualities are helpful. Wood and Garven recommend that interviewers have experience working with children, previous training in interviewing or counseling, a master's level education, the ability to establish rapport through warmth and friendliness, and the ability to take feedback constructively and change accordingly.

In addition to these characteristics, interviewer gender has also been examined as a factor related to disclosure in child sexual abuse cases. Lamb and Garretson (2003) reviewed 672 forensic interviews of children between ages 4 and 14 across Britain, Israel, and the United States. Their results showed that female interviewers asked significantly more suggestive questions with boys than with girls, while male interviewers did not show a distinction. In addition, girls provided significantly more details to female interviewers than male interviewers, while boys did not show a difference. Children between 4 and 6 years old gave more detailed responses to suggestive utterances made by interviewers of the opposite gender. These results suggest that the match between interviewer gender and child gender may have an important influence on disclosure.

While child gender is an important consideration for interviewers, age has been the most widely studied child characteristic influencing disclosure. Overall, younger children tend to provide fewer details and shorter responses during interviews than older children (e.g., Davies et al., 2000, Hershkowitz et al., 2002, Lamb \& Garretson, 2003, Sternberg et al., 1996 and Sternberg et al., 2001). In a study that included 142 forensic interviews with Israeli children ranging from 4 to 13 years old, Hershkowitz et al. (2002) found that 4- to 6-year-olds gave shorter responses and fewer details than older children in response to specific questions and invitations. However, in response to suggestive and option-posing questions, the youngest children gave significantly more details than older children. Davies et al. (2000) found similar results in their study of 36 videotaped interviews with children between age 4 and 14. Open-ended questions elicited longer and more accurate responses from 12- to 14-year-olds, while children between 4 and 11 years provided longer answers and more accurate information in response to closed questions and specific yet non-leading questions. Overall, length of responses significantly increased with age.

In both 1996 and 2001, Sternberg and colleagues found that younger children provided shorter and less detailed responses than older children. Invitations (i.e., questions or statements that prompt a response from the child) such as "What happened next?" and open-ended questions such as "Where were you when this happened?" were found to be much more effective with older children than with younger children. Finally, Lamb et al. (2003) examined forensic interviews of 130 children between 4 and 8 years old and found that older children provided significantly more details in response to invitations than younger children. The number of details elicited by invitations increased with age of the child. These studies highlight the importance of considering the child's age when choosing interviewing techniques (Carnes, 2000). In general, open-ended questions and invitations should be primarily used with older children and adolescents.

Age differences in disclosure are also likely to impact decisions regarding substantiation. Haskett, Wayland, Hutcheson, \& Tavana (1995) examined the factors involved in the decision to substantiate abuse across 175 child protective services (CPS) cases involving children between 2 and 19 years of age. These cases were handled by 20 different CPS workers across seven counties. This study found that cases with older children were more likely to be substantiated than those with younger children. The most important factors related to substantiation, as cited by CPS workers, were the degree of detail, consistency, and logic of the report. Considering the research showing that younger children provide less detail overall, this finding is not surprising. However, it underscores the need for effective interviewing techniques for use with young children.

Age differences can also be seen in the way disclosures are made. Campis, Hebden-Curtis, \& Demaso (1993) examined developmental differences between preschool children (ages 23 months to 6 years) and school age children 
(ages 7 to 17 years) in disclosures of sexual abuse. They found that preschool children tend to disclose in an accidental way, often following a triggering event, while school age children typically make intentional disclosures. Preschool children were also more likely to exhibit physical (i.e., abdominal pain, swelling, vaginal pain) and/or behavioral symptoms (i.e., nightmares, masturbation, aggression) than school age children. It may be helpful to consider these developmental differences when interviewing children of different ages. For example, when interviewing preschool children, physical and behavioral symptoms should be strongly considered in addition to verbal statements.

\section{Techniques used in forensic interviews}

While personal characteristics of the child and the interviewer may impact disclosure rates, specific interviewing techniques often play a greater role in disclosure. Several techniques have been examined in the literature, some of which appear to be very effective at eliciting detailed and accurate disclosures (e.g., Cantlon et al., 1996, Craig et al., 1999, Davies et al., 2000, Hewitt \& Arrowood, 1994, Huffman et al., 1999, Saywitz et al., 1992 and Wyatt, 1999). The focus of this discussion will be on techniques that are commonly used in forensic interviews and those with strong or mixed empirical support. These include allegation blind interviewing, open-ended questioning, cognitive interview techniques, truth-lie discussions, the Touch Survey, and anatomically detailed dolls.

\subsection{Allegation blind intervierws}

APSAC states that it is acceptable to gather information about the allegation before conducting the interview (APSAC, 2002). This information may be useful in orienting the interviewer and clarifying the child's statements. However, prior knowledge of allegations may increase interviewer bias and lead to suggestive and leading questioning (APSAC, 2002, Bruck \& Ceci, 1995, Cantlon et al., 1996 and Wyatt, 1999). Cantlon et al. (1996) compared allegation blind interviews (no information about allegations) to allegation informed interviews (prior information about allegations) across 1535 child sexual abuse cases over a 4-year period. In this study, higher disclosure rates were found with the allegation blind interview technique. The authors attributed this finding to increased attentiveness and patience on the part of the interviewer in allegation blind interviews, which likely increased rapport between the child and interviewer. In light of these findings and the higher perceived objectivity of allegation blind interviews in the courts (Cantlon et al., 1996), interviews should be allegation blind whenever possible. However, regardless of prior knowledge of the allegations, the interviewer should always take an objective and nonjudgmental stance toward the interview (APSAC, 2002, Bruck \& Ceci, 1995, Carnes, 2000, Lanning, 2002 and Saywitz et al., 2002).

\subsection{Open-ended questions}

Research has repeatedly shown that open-ended questions and invitations elicit longer, more detailed, and more accurate responses than other types of interviewer utterances in school age children and adolescents (Craig et al., 1999, Davies et al., 2000, Lamb \& Fauchier, 2001, Lamb \& Garretson, 2003 and Sternberg et al., 1996). However, as mentioned previously, this type of question is not as effective with very young children and often elicits shorter and less detailed responses than other types of interviewer utterances (Davies et al., 2000, Hershkowitz et al., 2002 and Sternberg et al., 1996). Lamb et al. (2003) examined 130 forensic interviews with children between 4 and 8 years old and found that nearly half of all information elicited from the children was in response to open-ended questions. Although older children provided more details overall, the proportion of details elicited by invitations and open-ended questions did not differ with age. However, cued invitations ("You mentioned that he touched you...tell me more about that") proved useful with younger children, particularly as a safer alternative than option-posing or closed questions.

Sternberg et al. (1996) examined 45 videotaped interviews with children ranging from 4 to 12 years old. This study found that invitations produced significantly more words and more details than focused types of utterances (i.e., directive, leading, suggestive), although this finding was much greater for older than for younger children. Overall, children's statements were three times richer in details and four times longer in response to open-ended or invitational 
questions than in response to focused questions. Open-ended questions may also be less likely to elicit self-contradictions in children's statements. Lamb and Fauchier (2001) examined 24 forensic interviews of seven children who were allegedly sexually abused in a daycare center and whose allegations led to convictions. The authors found that every self-contradiction that occurred was in response to a focused question. In contrast, no self-contradictions occurred in response to open-ended questions.

Craig et al. (1999) used Criteria-Based Content Analysis (CBCA) to assess the accuracy of children's statements in forensic interviews. CBCA is a procedure for rating the validity of children's statements based on 14 content criteria (e.g., quantity of details, logical structure). Their sample included 48 children, ranging in age from 3 to 16 years. Results of this study indicate that open-ended questions produced more free narrative responses and more accurate information than closed or direct questions. In contrast, direct questions were found to inhibit free narrative responses. A study by Davies et al. (2000) also used CBCA to assess the credibility of children's statements made in videotaped interviews conducted in England. Participants included 36 children between 4 and 14 years of age. They found that children between 12 and 14 years produced more accurate information (i.e., more $\mathrm{CBCA}$ criteria) and longer responses to open-ended questions than to other types of questions. However, specific yet non-leading questions elicited longer responses and more accurate information than other types of questions for children under age 12. In combination with research on young children's suggestibility (e.g., Bruck \& Ceci, 1995, Ceci \& Bruck, 1993 and Saywitz et al., 2002), these findings suggest overall that specific yet non-leading questions and cued invitations are most appropriate for young children, while open-ended questions should be used with school age children and adolescents.

\subsection{Cognitive interviewing}

In recent years, a set of four interviewing techniques known as the cognitive interview has been increasingly used in forensic interviews involving child sexual abuse cases (APSAC, 2002, Hayes \& Delamothe, 1997, Hershkowitz et al., 2002, Saywitz et al., 1992 and Saywitz et al., 2002). The cognitive interview was developed by Geiselman and colleagues in the 1980s for use with adult witnesses and victims (Geiselman et al., 1984). The techniques include mentally reconstructing the event (i.e., mental context reinstatement), reporting every detail of the event (regardless of perceived importance), recalling the event in different sequences, and describing the event from various perspectives (Fisher \& Geiselman, 1992 and Saywitz et al., 1992). In general, research has shown the cognitive interview to be effective in improving children's recall of events, although it appears to be more practical and effective with older children (e.g., APSAC, 2002, Hayes \& Delamothe, 1997 and Saywitz et al., 1992).

Saywitz et al. (1992) adapted the original cognitive interview for use with children. They also conducted a randomized controlled trial examining the utility of doing a practice cognitive interview about an unrelated innocuous event prior to interviewing the child about the event under investigation. The innocuous event involved an undergraduate research assistant dressed as a "surfer dude" introducing himself to the child participants in a waiting room. The event under investigation involved an argument over the use of a slide projector during a slide show witnessed by the child participants. Participants included 92 children between 8 and 12 years of age. Findings indicated that the cognitive interview was associated with $26 \%$ improvement in recalling correct facts over standard interviewing techniques. However, the practice interview was associated with 45\% improvement over standard interviewing techniques. Improvement was greater when all four cognitive techniques were used than when a subset was used, but each technique was also beneficial on its own. These results provide support for the use of the cognitive interview, but are limited by their lack of generalizability to child sexual abuse victims participating in forensic interviews.

Hayes and Delamothe (1997) examined effectiveness of two components of the cognitive interview (mental context reinstatement and reporting every detail) with 128 children ranging in age from 5 to 11 years. These components were chosen because they were seen as the most appropriate for use with children and had been shown in previous studies to be effective in isolation from other techniques. The other two components of the cognitive interview (i.e., recalling in different sequences, describing the event from different perspectives) are often very difficult for young children to perform. The cognitive interviewing techniques in this study significantly increased the amount of correct information recalled compared to standard interviewing techniques, even after controlling for other procedural differences. This finding was greater for older children than younger children and suggests that a subset of the cognitive interview may be a useful and practical alternative to the full cognitive interview. However, a small increase in confabulations during children's free recall was noted, indicating that caution may be necessary when using cognitive interviewing techniques. 
In a randomized controlled trial, Hershkowitz et al. (2002) compared one component of the cognitive interview, mental context reinstatement, to physical context reinstatement. Physical context reinstatement involved exposing an individual to the actual setting in which the event occurred (i.e., taking the child to the alleged crime scene). They examined 142 forensic interviews conducted in Israel with children between 4 and 13 years of age. Their study found that, in response to invitations, children in the mental context reinstatement group provided longer responses than children in the control group and the physical context reinstatement group, as well as more detailed responses than children in the physical context reinstatement group. These findings suggest that mental context reinstatement may be a useful component of the cognitive interview.

\subsection{Truth-lie discussions}

Interviewers often assess children's understanding of the difference between the "truth" and a "lie" before beginning the abuse-focused questioning. This discussion may demonstrate the child's competency and increase the credibility of his or her statements in court (APSAC, 2002 and Huffman et al., 1999). Wyatt (1999) recommends that children be asked if they have ever told a lie and what consequences result from telling lies. Wyatt also suggests that interviewers further test children's understanding of these concepts through the use of examples ("Tell me a lie about this chair"). APSAC also recommends that interviewers use concrete examples during truth-lie discussions (APSAC, 2002). It is often useful to obtain a verbal agreement from the child to tell the truth throughout the interview (Huffman et al., 1999 and Talwar et al., 2002). Huffman et al. (1999) examined the impact of truth-lie discussions (TLD) on 67 young children's responses during interviews. The children were interviewed about a neutral staged event that occurred at school. The study compared the effects of a control condition (no truth-lie discussion) to a standard truth-lie discussion and one that had been extended to include questions about the consequences of lying. Findings revealed no differences between the control group and the standard TLD group, while more accurate reports were made by children in the extended TLD group. These results suggest that it is important to include questions in the truth-lie discussion about the moral consequences of lying.

\subsection{Touch survey}

Another interviewing technique that has gained popularity in recent years is the Touch Survey, developed by Sandra Hewitt in the early 1980s (Carnes, 2000, Hewitt, 1998 and Hewitt \& Arrowood, 1994). It was developed as a screening for child abuse and was based on the idea that touches fall along a continuum, ranging from good to neutral to bad (Hewitt, 1998). Because preschool children often lack self-representational skills, Hewitt recommends that the Touch Survey be used with children over 3 years of age. Children between 4 and 8 years old are first given a warm-up exercise that involves reviewing various feelings and the faces associated with each. This exercise is intended to assess the child's self-representational skills, build rapport, and assess their attention span. The warm-up exercise is not necessary for children over 8 years old. The Touch Survey itself includes a discussion of various touches the child has experienced (i.e., hugging, kissing, hitting, sexual touches), feelings associated with the touches, locations on their body where they have received the touches, and who gave them the touches. Hewitt and Arrowood (1994) conducted a pilot study comparing the results from the Touch Survey to the results of complete case investigations for 42 children between the ages of 4 and 8 years. Findings revealed that none of the children claimed that abuse had occurred when the full evaluation determined it had not occurred (no false positives were found). However, $29 \%$ of the children did not disclose that abuse had occurred when the full evaluation determined that it had occurred. Therefore, the Touch Survey appears to err on the side of fewer but more accurate disclosures. This suggests that the Touch Survey is likely to be a useful tool, but should be used in combination with other empirically supported interviewing techniques. Further research is needed by individuals other than the author to determine its utility across settings.

\subsection{Anatomically detailed dolls}

One of the most controversial interviewing techniques discussed in the literature is the use of anatomically detailed dolls. While some claim they are useful in helping children to remember and describe the details of the abuse (APSAC, 2002, Boat \& Everson, 1996, Britton \& O’Keefe, 1990, Carnes, 2000 and Melton et al., 1997), 
others argue that they may decrease the quality of children's responses and can elicit sexual play even from nonabused children (Bruck \& Ceci, 1995, Ceci \& Bruck, 1993, DeLoache, 1993 and Santtila et al., 2004). Ceci and Bruck (1993) interviewed 3-year-old children using anatomically detailed dolls immediately after visiting their pediatrician. Half of the children received a genital examination and half of them did not, although $55 \%$ of the children who did not receive the examinations falsely reported that they received genital exams when they were interviewed using the dolls. A study by DeLoache (1993) involved interviews of 2- to 4-year-old children using dolls. This study found that preschoolers were more accurate in their reports when dolls were not used than when they were used.

Santtila et al. (2004) examined 27 transcribed forensic interviews conducted in Finland and found that interviews in which anatomically detailed dolls were used included more suggestive utterances and less detailed responses by the children. Another study by Britton and O'Keefe (1990) compared anatomically detailed dolls to nonanatomically detailed dolls across 136 forensic interviews in child sexual abuse cases and found no differences between groups in children's behavior with the dolls. However, results of this study were limited in that subjects were not randomized into groups, the primary investigator conducted all interviews herself, and children using nonanatomical dolls were allowed to choose from a selection of popular brand-name dolls. Overall, research in this area indicates that anatomically detailed dolls should be avoided with preschool children, due to the suggestibility and lack of self-representational skills found in this age group. They may be useful tools with school age children, but should be used with caution and only when necessary to facilitate communication (APSAC, 2002 and Carnes, 2000).

\section{New directions in forensic interviewing}

\subsection{Structured interviews}

While current techniques are continuously being examined through research and updated as needed, there are a few novel directions in which the field appears to be headed. A promising new approach to forensic interviews in child sexual abuse cases is the use of structured interviews, in which the interviewer utilizes a specific interviewing format (e.g., Orbach et al., 2000, Sternberg et al., 2001, Wells et al., 1997 and Wood \& Garven, 2000). Benefits of using a structured approach include limited training requirements, user-friendly and flexible protocols, past evidence that structured interviews are effective (i.e., Structured Clinical Interview for the DSM-IV), and improvement in quality of interviews (Wood \& Garven, 2000). Two examples of structured interviews intended for use with child sexual abuse victims are the Structured Interview of Symptoms Associated with Sexual Abuse (SASA) and the National Institute of Child Health and Human Development (NICHD) structured interview protocol (e.g., Orbach et al., 2000 and Wells et al., 1997).

The SASA was developed by Robert Wells and colleagues to be used as a structured interview with the alleged victim's parents (Wells et al., 1997). This interview is based on research findings regarding emotional, behavioral, and physical symptoms commonly associated with sexual abuse. It involves 26 areas of questioning, covering symptoms such as nightmares, difficulty concentrating, frequent stomachaches, increased knowledge about sex, aggression, seductive behavior towards others, and bedwetting. Wells (1992) examined the test-retest reliability of the SASA with 39 school age females undergoing sexual abuse evaluations. Average test-retest reliability for the full interview was found to be $74 \%$, while the test-retest reliability of individual items ranged from $48 \%$ to 94\%. Utility of the SASA was later examined for boys between the ages of 3 and 15 years (Wells et al., 1997). This study included 121 boys who were divided into a substantiated sexually abused group, an alleged abuse group, and a nonabused group. The authors found statistically significant differences between groups, with higher rates of symptoms in the sexually abused group and overall internal consistency of .83. Based on the results, the authors developed an Abbreviated SASA, consisting of the 12 items that were found to be significantly different between groups. This version demonstrated a specificity of $88 \%$ and sensitivity of $91 \%$. Though more research is needed, preliminary findings suggest that the SASA may be a useful tool for interviewing parents in child sexual abuse cases.

The NICHD investigative protocol was published in 2000 "to translate professional recommendations into everyday practice in the field” (Lamb \& Fauchier, 2001, p. 998). It was developed by Yael Orbach and colleagues based on research regarding effective interviewing techniques (Orbach et al., 2000). The NICHD protocol begins with 
an introduction, truth-lie discussion, and establishment of ground rules for the interview. Next, the interviewer focuses on building rapport and asks the child to describe a neutral event. The interviewer then transitions into the abuse-specific questioning by asking the child to describe why they are being interviewed. The interviewer is instructed to use nonsuggestive invitations and open-ended questions as much as possible, followed by focused nonsuggestive questions and option-posing questions if necessary. Each incident of possible abuse is examined in this way. Interviewers using the NICDH protocol also receive individual feedback and are required to attend regular group sessions to discuss interviews.

Several studies have demonstrated the NICHD protocol's effectiveness in reducing leading and suggestive questioning, increasing the use of open-ended questions, and increasing the number of details elicited from children (e.g., Lamb \& Garretson, 2003, Lamb et al., 2002, Orbach et al., 2000 and Sternberg et al., 2001). Orbach et al., 2000 compared 55 interviews in which the NICHD protocol was used to 50 interviews in which it was not used. They found that interviews using the protocol contained more open-ended questions and elicited more details from children than the non-protocol interviews. Sternberg et al. (2001) also compared 50 interviews using the NICHD protocol to 50 interviews conducted before the protocol was introduced. Results showed that NICHD interviews included 3 times more open-ended questions and significantly fewer suggestive and option-posing questions than non-protocol interviews. Furthermore, children interviewed with the NICHD protocol provided significantly more details overall and the protocol was found to be equally effective for all ages.

A study by Lamb et al. (2003) utilized the NICHD protocol during interviews of 130 children conducted in the United Kingdom and the United States. They also found no significant differences across age groups in interviewer utterances. This could be a positive finding, in that interviewers are not asking more suggestive questions to younger children than older children. However, it could also be a negative finding, based on the research that suggests interviewing techniques should be tailored to the age of the child (e.g., Davies et al., 2000, Hershkowitz et al., 2002, Lamb et al., 2003, Sternberg et al., 1996 and Sternberg et al., 2001). Lamb et al. (2002) examined necessity of requiring interviewers using the NICHD protocol to participate in ongoing intensive feedback. Participants included 74 children between 4 and 12 years old who were interviewed about sexual abuse allegations. Findings revealed that interview quality decreased dramatically when ongoing supervision ended. The proportion of suggestive and option-posing questions increased significantly and fewer details were elicited from children with interviewers who were not receiving supervision. This finding suggests that ongoing supervision and feedback are necessary components of the NICHD structured interview process.

\subsection{Extended forensic evaluation}

In addition to structured interviews, another promising development in the area of forensic interviewing is the extended forensic evaluation model. It has been suggested that multiple interviews are often necessary due to young children's brief attention spans, the discomfort they may feel in disclosing to a stranger, need for rapport in eliciting a disclosure, and utility of assessing the consistency of children's reports (APSAC, 2002, Carnes, 2000, Haskett et al., 1995 and Hewitt, 1998). The extended forensic evaluation model was developed by Connie Carnes at the National Children's Advocacy Center in Hunstville, Alabama to address the problem of children who do not disclose abuse during the first interview, but whose cases include other indicators that abuse has occurred (Carnes, 2000 and Carnes, 2005). During a two-year pilot study, 26\% of cases fit this description (Carnes, 2000 and Carnes et al., 1999). Children may also be referred for an extended forensic evaluation if information from the initial interview requires clarification or if the extent of the abuse is not disclosed during the initial interview (Carnes, 2000, Carnes, 2005 and Carnes et al., 1999). Goals of the extended forensic evaluation are to allow the child to disclose over time in a non-threatening environment, to determine if abuse has occurred and by whom, and to gather information to assist in legal and treatment decision-making (Carnes, 2000 and Carnes, 2005). Carnes (2000) recommends that interviewers should be graduate level mental health professionals who have previous experience working with children, training in child sexual abuse and child development, and experience conducting forensic interviews and testifying in court.

The structure of the extended forensic evaluation model includes five stages of information-gathering (Carnes, 2000 and Carnes, 2005). During the first stage, the interviewer gathers background information on the case from law enforcement and child protective services, medical information from physicians, and an interview is conducted with the non-offending caregiver. The second stage focuses on rapport-building, developmental assessment, and establishing ground rules for the interview process. In the third stage, social and behavioral assessments are conducted and 
behavioral checklists (i.e., Child Behavior Checklist, Trauma Symptom Checklist for Children, Child Sexual Behavior Inventory) are reviewed. The fourth stage consists of abuse-specific questioning, incorporating the use of various techniques, including open-ended questions, the Touch Survey, cognitive interviewing techniques, freestyle drawings, and nonanatomical dolls if necessary. Finally, during the fifth stage, the interviewer reviews and clarifies the child's statements, provides body safety information, and makes treatment referrals if necessary. The interviewer then uses the Forensic Evaluation Critical Analysis Guide (Carnes, 2000) to assess all of the information that has been gathered and to prepare a written report for the multidisciplinary team.

Though research is limited on this model, Carnes and colleagues have examined the effectiveness of the extended forensic evaluation on a few occasions (Carnes et al., 1999 and Carnes et al., 2001). Carnes et al. (1999) evaluated 51 children ages 2 to 16 using the extended forensic evaluation model and found that in $77 \%$ of cases, a clear determination was made regarding the credibility of disclosures. Thus, in the majority of cases, the evaluation accomplished its purpose. Carnes et al. (2001) also examined interviews of 147 children across 12 states using the extended forensic evaluation model. They found that in $64 \%$ of cases, a clear determination was made regarding credibility. They also compared a 4-session condition to an 8 -session condition and found that $95 \%$ of new disclosures were obtained by the sixth session, suggesting that 6 sessions is ideal. They found no difference in age, race, and gender on outcomes. Based on these findings, the recommended length is six sessions, including one session with the non-offending caregiver and five weekly 50-min sessions with the child (Carnes, 2000).

The extended forensic evaluation model appears to be a promising alternative for the subset of children who do not disclose in the first interview. However, several concerns with this model have been noted (e.g., APSAC, 2002, Bruck \& Ceci, 1995, Carnes, 2000, Santtila et al., 2004 and Wyatt, 1999). Extending the interview process over several sessions could potentially pose a risk to the child's safety. Sending a child home after the first or second session to a potentially abusive household and waiting a full week to conduct the next interview may put the child at risk for further abuse. In an ideal situation, a full disclosure would be obtained in the first interview and safety precautions could be taken immediately. Nevertheless, if the intention of the initial interview is to obtain a disclosure and this does not happen, the extended interview model appears to be the next best option. Another concern is related to the risks of repeated interviewing. Research has shown that repeated interviewing can lead to distortions in reporting, higher rates of self-contradictions, and increases in children's levels of distress (e.g., APSAC, 2002, Bruck \& Ceci, 1995 and Wyatt, 1999). In addition, a study by Santtila et al. (2004) examined 27 transcribed interviews conducted in Finland and found that significantly more new details were obtained in the first interview than in subsequent interviews and interviewers were more likely to use specific suggestive utterances in later interviews. However, these effects can likely be eliminated through training, supervision, and adherence to the protocol (APSAC, 2002 and Carnes, 2000).

A final criticism of the model is the need for separating clinical and forensic roles. Clinicians may use techniques that are beneficial in treatment, but that may hinder the investigation process (Carnes et al., 1999 and Wyatt, 1999). Forensic examiners and mental health professionals have very different goals when working with children who have made sexual abuse allegations (Carnes, 2000 and Wyatt, 1999). The goal of the forensic examiner is to obtain accurate information, while the goal of the mental health professional is to encourage the child to express his or her feelings and thoughts, regardless of their accuracy. For this reason, it is important that forensic examination be separated from therapy (Carnes, 2000 and Wyatt, 1999). The extended forensic evaluation model addresses this concern through rigorous training of forensic interviewers, requiring interviewers to collaborate with an investigative team, and referring the child to a different therapist after the evaluation is completed (Carnes, 2000 and Carnes et al., 1999).

\subsection{Child Advocacy Center model}

While multiple interviews may be necessary for some children, it may be best to limit the number of interviews and the range of locations and interviewers involved. According to some estimates, the average child may be interviewed ten times before going to court (Wyatt, 1999). Repeated interviewing and repeatedly asking similar questions have both been associated with inaccurate reporting and recanting allegations, particularly if early interviews are conducted inappropriately (e.g., APSAC, 2002, Bruck \& Ceci, 1995, Santtila et al., 2004 and Wyatt, 1999). Furthermore, the child's suffering is exacerbated when they are repeatedly and unnecessarily subjected to stressful and upsetting interviews with multiple strangers. In response to this problem, the Child Advocacy Center (CAC) model was developed in Huntsville, Alabama in 1985. The goal of all Child Advocacy Centers is to "ensure that children are not further victimized by the intervention systems designed to protect them" (National Children's Advocacy Center, 2005a and National Children's Advocacy Center, 2005b). 
Accreditation, training, practice standards, and services for Child Advocacy Centers are provided by the National Children's Alliance, a nationwide non-profit organization (Murray, 2005). In 2004, the National Children's Alliance had 41 state chapters and 330 member centers (National Children's Alliance, 2003). Approximately 124,900 children were served by Child Advocacy Centers in 2003 alone. Though the majority of cases seen at Child Advocacy Centers involve sexual abuse ( $73 \%$ in 2003), cases involving physical abuse, neglect, domestic violence, and other forms of abuse are also seen (National Children's Alliance, 2003).

Child Advocacy Centers are safe, neutral, child-friendly facilities where children and families can receive a range of services. These include forensic interviews conducted by trained interviewers, medical examinations, mental health services, victim support and advocacy, case review by the multidisciplinary team, and tracking of case progress and outcomes. In addition, Child Advocacy Centers provide specialized training and support for professionals in the community and strive to enhance community awareness of child abuse (Murray, 2005, National Children's Advocacy Center, 2005a, National Children's Advocacy Center, 2005b and National Children's Alliance, 2003). The CAC model is based on a multi-disciplinary approach to child abuse cases. This approach is beneficial because it is in the best interests of the child, reduces the number of interviews, provides the victim with support, promotes understanding of other disciplines, increases access to training opportunities, and leads to better informed decisions (APSAC, 2002, Lanning, 2002, National Children's Advocacy Center, 2005a and National Children's Advocacy Center, 2005b). Professionals from various disciplines (i.e., law enforcement, mental health, prosecution, medicine, child protection, victim advocacy) coordinate their efforts and work together to make team decisions. Communities with Child Advocacy Centers are believed to have more efficient referrals to physicians and mental health professionals, fewer child interviews, and more efficient follow-up procedures than communities without them (National Children's Advocacy Center, 2005a and National Children's Advocacy Center, 2005b). For these reasons, the Child Advocacy Center model appears to be a commendable model for addressing child sexual abuse allegations.

\section{Implications for research and practice}

Several limitations were found in the research reviewed in this paper. First, studies examining interviewing techniques tended to use a wide variety of definitions for various types of interviewer utterances (e.g., Craig et al., 1999, Davies et al., 2000, Lamb \& Fauchier, 2001, Lamb et al., 2003, Santtila et al., 2004, Sternberg et al., 1996 and Sternberg et al., 2001). Some studies included invitations and open-ended questions in the same category (e.g., Craig et al., 1999, Davies et al., 2000 and Lamb \& Fauchier, 2001), while others examined one or the other alone (e.g., Lamb et al., 2003 and Santtila et al., 2004). The terms "open-ended questions" and "directive utterances" were at times used interchangeably (e.g., Lamb et al., 2003), while at other times "directive utterances" was used to describe questions which limited the child's responses (Craig et al., 1999). The confusion over definitions and names of interviewer utterances may have hindered interpretation of research findings. Future studies should adhere to an agreed-upon coding scheme, such as that outlined by Lamb and colleagues or guidelines such as the Memorandum of Good Practice in England (e.g., Davies et al., 2000 and Lamb et al., 1996).

A second area of limitation was that much of the research on certain interviewing techniques (i.e., Touch Survey, NICHD structured protocol, SASA, extended forensic evaluation model) was limited to the developers of these techniques. Few studies have been conducted by researchers who were not involved in the development process, leaving the readers unable to draw conclusions regarding the effectiveness of these techniques. Therefore, more research is needed by individuals who are unrelated to the development process. Third, while several of the studies discussed in this paper included adolescents in their samples (e.g., Carnes et al., 1999, Carnes et al., 2001, Craig et al., 1999, Davies et al., 2000, Hershkowitz et al., 2002, Lamb \& Garretson, 2003 and Wells et al., 1997), very little research has focused on adolescents alone. Future research should be conducted using samples of adolescents and examining issues specific to adolescents in relation to forensic interviewing.

A fourth area of limitation involved outcome variables used in these studies. In much of the research reviewed here, the investigators were unable to know for certain if the abuse allegations were true. As a result, they relied on other variables (i.e., absence of self-contradictions, number of details elicited, length of child responses) to determine the effectiveness of various interviewing techniques (e.g., Lamb et al., 2003, Santtila et al., 2004, Sternberg et al., 1996 and Sternberg et al., 2001). While this is often necessary when conducting research in the field, it is certainly not ideal. More research is needed using samples of children for which abuse allegations have been substantiated. The use of Criteria-Based Content Analysis (CBCA) is also a promising solution to this problem (Craig et al., 1999 and Davies et al., 2000). As mentioned previously, CBCA is an empirically based procedure for rating children's statements during forensic interviews. The 14 content criteria used to assess 
the accuracy of children's statements have been shown to successfully discriminate accurate from inaccurate abuse allegations (Craig et al., 1999). This appears to be a useful outcome variable for use in research related to forensic interviewing.

Despite the above-mentioned limitations, research in the area of forensic interviewing provides a basis for several recommendations. The following recommendations for forensic interviewers are empirically derived and based on the information in this literature review.

1. Whenever possible, interviews should be conducted in a safe, neutral, and preferably child-friendly environment, such as a Child Advocacy Center (e.g., APSAC, 2002, Carnes, 2000, Lanning, 2002, National Children's Advocacy Center, 2005a, National Children's Advocacy Center, 2005b and National Children's Alliance, 2003).

2. A multidisciplinary approach to child abuse investigations is preferable when the option is available (e.g., APSAC, 2002, Carnes, 2000, Lanning, 2002, National Children's Advocacy Center, 2005a and National Children's Advocacy Center, 2005b).

3. The child's age should be considered when choosing interviewing techniques. Open-ended questions should be used with older children when possible, while cued invitations and specific yet non-leading questions should be used with younger children (Carnes, 2000, Davies et al., 2000, Hershkowitz et al., 2002, Lamb et al., 2003, Sternberg et al., 1996 and Sternberg et al., 2001). Leading and suggestive questions should always be avoided.

4. Interviewer gender should be considered when scheduling appointments and training new interviewers. Based on the findings of Lamb and Garretson (2003), it might be particularly helpful to pair female interviewers with female victims.

5. Forensic interviewers should possess the ability to establish rapport through warmth and friendliness, experience working with children, previous training in interviewing or counseling, training in child sexual abuse and child development, a master's level education, an objective and nonjudgmental stance toward interviews, and the ability to take feedback constructively and change accordingly (APSAC, 2002, Carnes, 2000 and Wood \& Garven, 2000).

6. Structured interview protocols (i.e., NICHD investigative interview) are recommended, due to their effectiveness, ease of use, and limited training requirements (Lamb \& Garretson, 2003, Lamb et al., 2002, Lamb et al., 2003, Orbach et al., 2000, Sternberg et al., 2001 and Wood \& Garven, 2000). However, they should be used in combination with ongoing supervision and feedback.

7. Ground rules should be outlined for the child at the onset of the interview, including what should happen if the child does not know an answer, does not understand the question, does not remember something, does not want to answer a question, or if the interviewer makes a mistake (e.g., APSAC, 2002 and Carnes, 2005).

8. Before discussing the abuse allegations, the interviewer should discuss with the child the difference between a truth and a lie, the consequences of telling a lie, and obtain the child's agreement to tell the truth (e.g., APSAC, 2002, Huffman et al., 1999, Talwar et al., 2002 and Wyatt, 1999).

9. The Touch Survey can be used as a technique to elicit details about good and bad touches that the child has experienced, although it should be used in combination with other empirically supported techniques (Carnes, 2000, Hewitt, 1998 and Hewitt \& Arrowood, 1994).

10. Cognitive interviewing techniques should be used whenever possible (particularly with older children) to obtain further details about the abuse (APSAC, 2002, Hayes \& Delamothe, 1997, Hershkowitz et al., 2002 and Saywitz et al., 1992). The child's developmental level should be considered when determining which techniques may be most useful (e.g., Hayes \& Delamothe, 1997).

11. Anatomically detailed dolls should be used cautiously, should be avoided with very young children, and should be introduced to obtain further details only after the child has already disclosed (e.g., APSAC, 2002, Bruck \& Ceci, 1995, Carnes, 2000, Ceci \& Bruck, 1993, DeLoache, 1993 and Santtila et al., 2004).

12. If conducted appropriately, extended forensic evaluation appears to be a valuable option for children who do not disclose during the initial interview and should be used only when necessary (Carnes, 2000, Carnes, 2005, Carnes et al., 1999 and Carnes et al., 2001).

Forensic interviewing in child sexual abuse cases has evolved greatly through the years. Research in the area has provided valuable information regarding effective and appropriate interviewing techniques. Though more research is needed to further explore these techniques, forensic interviewers can benefit considerably from the guidance that research provides. 


\section{References}

APSAC, 2002: American Professional Society on the Abuse of Children (APSAC), Practice guidelines: Inve stigative interviewing in cases of alleged child abuse (2002).

Boat \& Everson, 1996: B. Boat and M. Everson, Concerning practices of interviewing when using anatomical dolls in child protective services, Child Maltreatment 1 (1996), pp. 96-104.

Boney-McCoy \& Finkelhor, 1995: S. Boney-McCoy and D. Finkelhor, Prior victimization: A risk factor for child sexual abuse and for PTSD-related symptomatology among sexually abused youth, Child Abuse and Neglect 19 (1995), pp. 1401-1421.

Britton \& O'Keefe, 1990: H.L. Britton and M.A. O'Keefe, Use of nonanatomical dolls in the sexual abuse interview, Child Abuse and Neglect 15 (1990), pp. 567-573.

Bruck \& Ceci, 1995: M. Bruck and S.J. Ceci, Amicus brief for the case of State of New Jersey v. Michaels presented by Committee of Concerned Social Scientists, Psychology, Public Policy, and Law 1 (1995), pp. 272-322.

Campis et al., 1993: L.B. Campis, J. Hebden-Curtis and D.R. Demaso, Developmental differences in detection and disclosure of sexual abuse, Journal of the American Academy of Child and Adolescent Psychiatry 32 (1993), pp. 920-924.

Cantlon et al., 1996: J. Cantlon, G. Payne and C. Erbaugh, Outcome-based practice: Disclosure rates of child sexual abuse comparing allegation blind and allegation informed structured interviews, Child Abuse and Neglect 20 (1996), pp. 1113-1120.

Carnes, 2005: C. Carnes, Extended forensic evaluation (2005) [On-line]. Available: http://www.nationalcac.org/professionals/model/ forensic eval.html.

Carnes, 2000: C.N. Carnes, Forensic evaluation of children when sexual abuse is suspected, National Children's Advocacy Center, Huntsville, AL (2000).

Carnes et al., 2001: C.N. Carnes, D. Nelson-Gardell, C. Wilson and U.C. Orgassa, Extended forensic evaluation when sexual abuse is suspected: A multisite field study, Child Maltreatment 6 (2001), pp. 230-242.

Carnes et al., 1999: C.N. Carnes, C. Wilson and D. Nelson-Gardell, Extended forensic evaluation when sexual abuse is suspected: A model and preliminary data, Child Maltreatment 4 (1999), pp. 242-254.

Ceci \& Bruck, 1993: S.J. Ceci and M. Bruck, Children's recollections: Translating research into policy. SRCD Social policy reports (1993).

Craig et al., 1999: R.A. Craig, R. Scheibe, D.C. Raskin, J.C. Kircher and D.H. Dodd, Interviewer questions and content analysis of children's statements of sexual abuse, Applied Developmental Science 3 (1999) (2), pp. 77-85.

Davies et al., 2000: G.M. Davies, H.L. Westcott and N. Horan, The impact of questioning style on the content of investigative interviews with suspected child sexual abuse victims, Psychology, Crime, and Law 6 (2000), pp. 81-97.

DeLoache, 1993: J.S. DeLoache, The use of dolls in interviewing young children. In: M.S. Zaragoza, J.R. Graham, G.C.N. Hall, R. Hirschman and Y.S. Ben-Porath, Editors, Memory and testimony in the child witness, Sage, Thousand Oaks, CA (1993).

Fisher \& Geiselman, 1992: R.P. Fisher and R.E. Geiselman, Memory-enhancing techniques for investigating interviewing: The cognitive interview, Charles C. Thomas, Springfield, IL (1992).

Geiselman et al., 1984: R.E. Geiselman, R.P. Fisher, I. Firstenberg, L.A. Hutton, S. Sullivan and I. Avetissian et al., Enhancement of eyewitness memory: An empirical evaluation of the cognitive interview, Journal of Police Science and Administration 12 (1984), pp. 74-80.

Haskett et al., 1995: M.E. Haskett, K. Wayland, J.S. Hutcheson and T. Tavana, Substantiation of sexual abuse allegations: Factors involved in the decision-making process, Journal of Child Sexual Abuse 4 (1995) (2), pp. 19-44.

Hayes \& Delamothe, 1997: B.K. Hayes and K. Delamothe, Cognitive interviewing procedures and suggestibility in children's recall, Journal of Applied Psychology 82 (1997), pp. 562-577.

Hershkowitz et al., 2002: I. Hershkowitz, Y. Orbach, M. Lamb, K.J. Sternberg and D. Horowitz, A comparison of mental and physical context reinstatement in forensic interviews with alleged victims of sexual abuse, Applied Cognitive Psychology 16 (2002), pp. 429-441.

Hewitt, 1998: S.K. Hewitt, Assessing allegations of sexual abuse in preschool children: Understanding small voices, Sage Publications, Thousand Oaks, CA (1998).

Hewitt \& Arrowood, 1994: S.K. Hewitt and A.A. Arrowood, Systematic touch exploration as a screening procedure for child abuse: A pilot study, Journal of Child Sexual Abuse 3 (1994) (2), pp. 31-43.

Hsu et al., 2002: E. Hsu, G. Sedlar, M.F. Flood and D.J. Hansen, Child sexual abuse. In: M. Hersen, Editor, Clinical behavior therapy: Adults and children, Wiley, New York (2002), pp. 449-473.

Huffman et al., 1999: M.L. Huffman, A.R. Warren and S.M. Larson, Discussing truth and lies in interviews with children: Whether, why, and how?, Applied Developmental Science 3 (1999), pp. 6-15. 
Lamb \& Fauchier, 2001: M.E. Lamb and A. Fauchier, The effects of question type on self-contradictions by children in the course of forensic interviews, Applied Cognitive Psychology 15 (2001), pp. 483-491.

Lamb \& Garretson, 2003: M.E. Lamb and M.E. Garretson, The effects of interviewer gender and child gender on the informativeness of alleged child sexual abuse victims in forensic interviews, Law and Human Behavior 27 (2003) (2), pp. 157-171.

Lamb et al., 1996: M.E. Lamb, I. Hershkowitz, K.J. Sternberg, P.W. Esplin, M. Hovav and T. Manor et al., Effects of investigative utterance types on Israeli children's responses, International Journal of Behavioural Development 19 (1996), pp. 627-637.

Lamb et al., 2002: M.E. Lamb, K.J. Sternberg, Y. Orbach, P.W. Esplin and S. Mitchell, Is ongoing feedback necessary to maintain the quality of investigative interviews with allegedly abused children?, Applied Developmental Science 6 (2002), pp. 35-41.

Lamb et al., 2003: M.E. Lamb, K.J. Sternberg, Y. Orbach, P.W. Esplin, H. Stewart and S. Mitchell, Age differences in young children's responses to open-ended invitations in the course of forensic interviews, Journal of Consulting and Clinical Psychology 71 (2003) (5), pp. 926-934.

Lanning, 2002: K. Lanning, Criminal investigation of sexual victimization of children. In: J.E.B. Myers, L. Berliner, J. Briere, C.T. Hendrix, C. Jenny and T.A. Reid, Editors, The APSAC handbook on child maltreatment (Second edition), Sage Publications, Inc., Thousand Oaks, CA (2002), pp. 329-347.

Melton et al., 1997: G.B. Melton, J. Petrila, N.G. Poythress and C. Slobogin, Psychological evaluations for the courts: A handbook for mental health professionals and lawyers (2nd ed.), Guilford Press, New York (1997).

Murray, 2005: B. Murray, National Children's Alliance standards for full member programs (2005) Online: http://www.nca-online. org/network.html.

National Children's Advocacy Center, 2005a: National Children's Advocacy Center, Child forensic interview model (2005). Online: http://www.nationalcac.org/professionals/model/forensic_interview.html.

National Children's Advocacy Center, 2005b: National Children's Advocacy Center, The CAC model. Online: http://www.nationalcac.org/professionals/model/cac_model.html.

National Children's Alliance, 2003: National Children's Alliance, Joining hands to protect children: National Children's Alliance 2003 annual report, Author, Washington, DC (2003).

Orbach et al., 2000: Y. Orbach, I. Hershkowitz, M.E. Lamb, P.W. Esplin and D. Horowitz, Assessing the value of structured protocols for forensic interview of alleged child abuse victims, Child Abuse and Neglect 24 (2000), pp. 733-752.

Santtila et al., 2004: P. Santtila, J. Korkman and N.K. Sandnabba, Effects of interview phase, repeated interviewing, presence of a support person, and anatomically detailed dolls on child sexual abuse interviews, Psychology, Crime, and Law 10 (2004), pp. $21-35$.

Saywitz et al., 1992: K.J. Saywitz, R.E. Geiselman and G.K. Bornstein, Effects of cognitive interviewing and practice on children's recall performance, Journal of Applied Psychology 77 (1992), pp. 744-756.

Saywitz et al., 2002: K.J. Saywitz, G.S. Goodman and T.D. Lyon, Interviewing children in and out of court: Current research and practice implications. In: J.E.B. Myers, L. Berliner, J. Briere, C.T. Hendrix, C. Jenny and T.A. Reid, Editors, The APSAC handbook on child maltreatment (Second edition), Sage Publications, Inc., Thousand Oaks, CA (2002), pp. 329-347.

Sternberg et al., 1996: K.J. Sternberg, M.E. Lamb, I. Hershkowitz, P.W. Esplin, A. Redlich and N. Sunshine, The relation between investigative utterance types and the informativeness of child witnesses, Journal of Applied Developmental Psychology 17 (1996) (3), pp. 439-451.

Sternberg et al., 2001: K.J. Sternberg, M.E. Lamb, Y. Orbach, P.W. Esplin and S. Mitchell, Use of a structured investigative protocol enhances young children's responses to free-recall prompts in the course of forensic interviews, Journal of Applied Psychology 86 (2001), pp. 997-1005.

Talwar et al., 2002: V. Talwar, K. Lee, N. Bala and R.C.L. Lindsay, Children's conceptual knowledge of lying and its relation to their actual behaviors: Implications for court competence examines, Law and Human Behavior 26 (2002), pp. 395-415.

Tyler, 2002: K.A. Tyler, Social and emotional outcomes of childhood sexual abuse: A review of recent research, Aggression and Violent Behavior 7 (2002), pp. 567-589.

U.S. Department of Health and Human Services, 2005: U.S. Department of Health and Human Services, Child maltreatment 2003, U.S. Government Printing Office, Washington, DC (2005).

Wells, 1992: Wells, R.D. (1992). Test retest reliability of the structured interview for symptoms associated with sexual abuse (SASA). Unpublished manuscript, Department of Pediatrics, Valley Medical Center, Fresno, CA.

Wells et al., 1997: R. Wells, J. McCann, J. Adams, J. Voris and B. Dahl, A validational study of the structured interview of symptoms associated with sexual abuse (SASA) using three samples of sexually abused, allegedly abused, and nonabused boys, Child Abuse and Neglect 21 (1997), pp. 1159-1167.

Wood \& Garven, 2000: J.M. Wood and S. Garven, How sexual abuse interviews go astray: Implications for prosecutors, police, and child protection services, Child Maltreatment 5 (2000), pp. 109-118. 